\title{
The importance of disease associations and concomitant therapy for the long-term management of psoriasis patients
}

\author{
Ulrich Mrowietz • James T. Elder • Jonathan Barker
}

Received: 26 July 2006 / Revised: 31 August 2006 / Accepted: 4 September 2006 / Published online: 5 October 2006

(C) Springer-Verlag 2006

\begin{abstract}
It is well established that several inflammatory-type conditions, such as arthritis, diabetes, cardiovascular disease, and irritable bowel disease exist comorbidly and at an increased incidence in patients with psoriasis. Psoriasis and other associated diseases are thought to share common inflammatory pathways. Conditions such as these, with similar pathogenic mechanisms involving cytokine dysregulation, are referred to as immune-mediated inflammatory diseases (IMIDs). Considerable evidence for the genetic basis of cormobidities in psoriasis exists. The WHO has reported that the occurrence of chronic diseases, including IMIDs, are a rising global burden. In addition, conditions linked with psoriasis have been associated with increasing rates of considerable morbidity and mortality. The presence of comorbid conditions in psoriasis patients has important implications for clinical management. QoL, direct health care expenditures and pharmacokinetics of concomitant therapies are
\end{abstract}

The authors are consultants to Schering-Plough Pharmaceuticals and have received an honorarium for their participation in this programme.

U. Mrowietz $(\bowtie)$

Department of Dermatology, University of Kiel,

Schittenhelmstr. 7, 24105 Kiel, Germany

e-mail: umrowietz@dermatology.uni-kiel.de

J. T. Elder

Department of Dermatology and Radiation Oncology,

University of Michigan Medical School,

3312 CCGC Box 0932, Ann Arbor, MI 48109, USA

J. Barker

St. John's Institute of Dermatology, St. Thomas' Hospital,

Lambeth Palace Rd, London SE1 7EH, UK impacted by the presence of comorbid conditions. For example, methotrexate is contraindicated in hepatic impairment, while patients on ciclosporin should be monitored for kidney function. In addition, some agents, such as beta blockers, lithium, synthetic antimalarial drugs, NSAIDs and tetracycline antibiotics, have been implicated in the initiation or exacerbation of psoriasis. Consequently, collaboration between physicians in different specialties is essential to ensuring that psoriasis treatment benefits the patient without exacerbating associated conditions.

Keywords Psoriasis · Comorbid · Quality-of-life · Biologics $\cdot$ Inflammation

\section{Psoriasis: from gene to clinic}

A satellite symposium held at the Royal College of Physicians, London, United Kingdom, in December 2005 investigated the associations between psoriasis, comorbid diseases and concomitant therapy and their significance for long-term patient treatment. The programme generated substantial debate and raised some clinically significant issues, which are further outlined here.

\section{Introduction}

Psoriasis is a common, chronic, immune-mediated inflammatory disease with a recognised genetic predisposition. It has been observed that, while the frequency of skin conditions such as acne, urticaria and atopic dermatitis are reduced in patients with psoriasis 
compared with expected frequencies in the general population, the frequency of some noncutaneous, although related, conditions is significantly increased $[26,53]$. Perhaps the best known noncutaneous condition associated with psoriasis is joint disease, mostly expressed as psoriatic arthritis (PsA). In hospitalised psoriasis patients, a diagnosis of PsA [according to the European Spondyloarthropathy Study Group (ESSG) criteria] was present in $7.7 \%$ (71/936) of patients; however, many more patients also reported paresthesias $(12.3 \%)$, arthralgia $(7 \%)$, stiffness $(4.2 \%)$, swelling $(3.7 \%)$ and ankylosis (1.2\%) without meeting all of the ESSG criteria for PsA [19]. Further estimates place the occurrence of PsA in patients with psoriasis at upwards of $30 \%$ [31]. An additional study by Zachariae et al. [83] established even higher rates for psoriasis with joint involvement in 5,795 members of psoriasis associations from the Nordic countries and 702 patients seen by Nordic dermatologists. The result showed that upwards of $30 \%$ of all patients who presented with psoriasis were also diagnosed with arthritis by their dermatologist or a rheumatologist. In a recent study in a geographically defined population in Norway Madland et al. [41] found a prevalence of PsA in 2 per 1,000 inhabitants, which corresponds to about $10 \%$ in patients with psoriasis assuming a prevalence of psoriasis of $2 \%$.

These results demonstrate that the prevalence of arthritis in patients with psoriasis may actually be higher than the previously accepted rate of 7\% [83]. Inflammatory bowel disease is also more frequently observed in patients with psoriasis. An increased relative risk for Crohn's disease (CD) of 2.9 was found to be associated with a prior diagnosis of psoriasis in a Swedish case-control study [54]. Conversely, patients with $\mathrm{CD}$ are about seven times more likely to develop psoriasis than controls [5, 37, 82].

Analysis of data from more than 40,000 dermatological inpatients with psoriasis compared with control patients with nonpsoriatic skin diseases (adjusting for age and gender) revealed high ratios of observed versus expected frequencies for obesity $(2.05, P<0.05)$, hypertension $(1.90, P<0.01)$, heart failure $(1.83, P<$ $0.001)$ and diabetes mellitus $(1.47, P<0.05)$ [26]. An additional analysis (retrospective chart review, 19972000) of 753 patients from an academic dermatology practice also confirms the presence of comorbidities in patients with psoriasis [53]. In fact, a comorbid diagnosis was confirmed in 551 patients $(73 \%)$, with hypertension, dyslipidemia, diabetes and heart disease being the most common comorbidities identified [53]. Furthermore, patients with psoriasis appear to be at increased risk of atherosclerosis, which may at least in part be explained by an abnormal plasma lipid metabolism; serum lipoprotein (a) and triglycerides are significantly increased in patients with psoriasis compared with healthy control subjects [73], while high-density lipoprotein (HDL) cholesterol is significantly decreased [60]. Indeed, in patients with PsA, alterations of the lipid profile suggest similarities to the diabetic lipid profile, which is known to substantially increase cardiovascular risk [30].

Nearly 30 years ago it was reported that the rates of occlusive vascular disease were significantly higher in dermatological patients with psoriasis than those without and that the risk was greater in patients with predisposing factors [44]. Metabolic syndrome has since been identified as a clustering of metabolic abnormalities in individuals and is associated with a threefold increase in type 2 diabetes and a twofold increase in cardiovascular disease [86]. The most recent definition of metabolic syndrome requires central obesity (defined as body mass index $>30 \mathrm{~kg} / \mathrm{m}^{2}$ ) plus any two of the following: elevated plasma triglycerides, reduced HDL cholesterol, elevated blood pressure and raised fasting plasma glucose (Table 1). Such clustering of risk factors has been observed in patients with psoriasis [26].

Psoriasis, especially in patients with more severe disease, has also been associated with other factors that can increase cardiovascular risk, thereby increasing morbidity and mortality. Alcoholism and liver cirrhosis are reportedly more common in psoriasis [39] with a reported prevalence of alcoholism of $18 \%$ among psoriatic patients compared with $2 \%$ in other dermatologic controls [22, 46]. Cigarette smoking has also been associated with psoriasis. A recent case-control study revealed that the risk of psoriasis was higher in exsmokers and current smokers than in those who never smoked (odds ratios of 1.9 and 1.7, respectively), and smoking was strongly associated with the occurrence of pustular lesions (odds ratio $=5.3$ for smokers) [48]. In a cross-sectional study, patients with psoriasis that enrolled in the prospective Utah Psoriasis Initiative had a significantly higher prevalence of obesity and smoking than the general population of Utah $(P<0.001$ for both). The prevalence of obese smokers was significantly higher $(P<0.001)$ [27].

A number of psychiatric/psychological comorbidities have also been observed in patients with psoriasis [22]. An association between psoriasis and stressful life events in the year preceding diagnosis has been reported, suggesting that psychological stress may have a role in the pathogenesis of psoriasis [48]. Furthermore, psoriasis-related stress can play a role in the exacerbation of psoriasis, and greater stress reactivity has been associated with onset of psoriasis at an earlier 
Table 1 Diseases associated with psoriasis [65]

\begin{tabular}{llll}
\hline Condition & $\begin{array}{l}\text { Prevalence in } \\
\text { psoriasis } n(\%)\end{array}$ & $\begin{array}{l}\text { Hospital controls } \\
\text { OR }(95 \% \text { CI })\end{array}$ & RKI sample OR (95\% CI) \\
\hline Diabetes mellitus type I & $11(1.9)$ & $3.99(1.30-12.2)^{*}$ & $6.34(2.80-14.3)^{* * *}$ \\
Diabetes mellitus type II & $68(11.7)$ & $2.48(1.70-3.61)^{* *}$ & $2.07(1.50-2.85)^{* * *}$ \\
Arterial hypertension & $127(21.9)$ & $3.27(2.41-4.43)^{* * *}$ & $1.39(1.09-1.77)^{*}$ \\
Hyperlipoproteinemia & $30(5.2)$ & $2.09(1.23-3.54)^{* *}$ & $0.83(0.54-1.28)^{\mathrm{f}}$ \\
Coronary heart disease & $32(5.5)$ & $1.77(1.07-2.93)^{*}$ & $0.93(0.60-1.43)^{\mathrm{f}}$ \\
Metabolic syndrome & $25(4.3)$ & $5.92(2.78-12.8)^{* * *}$ & $2.20(1.41-3.43)^{* *}$ \\
Alcohol consumption none & $246(42.3)$ & $2.78(2.14-3.62)^{* * *}$ & $2.03(1.62-2.55)^{* * *}$ \\
$\quad$ vs. moderate & $75(12.9)$ & $3.33(2.20-5.05)^{* * *}$ & $2.00(1.45-2.77)^{* * *}$ \\
None vs. regularly & $24(4.1)$ & $3.61(1.85-7.07)^{* * *}$ & $8.50(5.28-16.8)^{* * *}$ \\
None vs. heavy & $264(45.4)$ & $2.96(2.27-3.84)^{* * *}$ & $2.49(2.00-3.10)^{* * *}$ \\
Cigarette smoking & & & \\
\hline
\end{tabular}

The prevalence of diseases in plaque-type psoriasis patients $(n=581)$ was compared with hospital-based controls $(n=1,044)$ and a population-based survey (RKI sample; $n=4,705$ ). Common odds ratios adjusted for age and sex are presented with their $95 \%$ confidence intervals $(95 \% \mathrm{CI}), f$ not significant

${ }^{a}$ Due to low numbers, only adjusted for sex

$* P<0.05$; ** $P<0.01 ; * * * P<0.0001$ by Mantel-Haenszel test

age [22]. High depression/anxiety scores, obsessionality and difficulties with verbal expression of emotions (especially anger) have been reported in various crosssectional surveys [22]. Severely affected psoriatic inpatients $(>30 \%$ body surface area affected) were likely to suffer clinical depression and suicidal ideation (7.2\%); however, suicidal ideation was still relatively high $(2.5 \%)$ in less severely affected outpatients $(<30 \%$ total body surface area affected) and comparable to the reported prevalence among general medical patients $(2.4-3.3 \%)$ [21].

These identified diseases associated with psoriasis have led clinicians to look at underlying mechanisms that might be involved and start considering how managing comorbid conditions may impact treatment selection and outcome for the psoriasis patient.

\section{Common inflammatory pathways in psoriasis and associated diseases}

The immune system has been strongly implicated in the pathogenesis of psoriasis that resembles a $\mathrm{T}$ cellmediated, autoimmune, inflammatory disease [7]. T cells are found in the dermis and epidermis and are accompanied by increased numbers of dermal dendritic cells, macrophages and mast cells [51]. It is thought that a stimulus (such as trauma or infection) triggers a plexus of cellular events by inciting a cascade of cytokines, creating an inflammatory response. Dendritic cells and $\mathrm{T}$ cells become activated with the formation of an immunological synapse-a multimolecular complex at the $\mathrm{T}$ cell-antigen-presenting cell interface that facilitates immune cell interactions. Once activated, dendritic antigen-presenting cells and $\mathrm{T}$ cells release cytokines, chemokines and growth factors, which trigger keratinocyte proliferation, altered differentiation and an angiogenic tissue response, giving rise to psoriatic lesions (Fig. 1).

Other inflammatory diseases, such as rheumatoid arthritis (RA) and CD, are thought to involve similar pathogenetic mechanisms. Recent research has shown that inflammation plays a key role in atherosclerosis [24]. A T-cell infiltrate is always present in atherosclerotic lesions, with activated $\mathrm{T}$ cells producing a type 1 helper (Th1) response. Psoriasis is up to seven times more common in patients with CD than in controls [37, 82] and, as discussed previously, there is often joint involvement in psoriasis. Such extracutaneous manifestations of the disease substantiate that psoriasis is a systemic disease and not just a skin disease [14].

The term immune-mediated inflammatory disease (I.M.I.D.) has been developed to describe conditions, such as psoriasis, CD and RA, with common inflammatory mechanisms described above involving cytokine dysregulation. This term also encompasses type 1 diabetes mellitus, ulcerative colitis, systemic lupus erythematosus, multiple sclerosis, ankylosing spondylitis and uveitis and may further be expanded to include asthma and atherosclerosis [79].

Atherosclerosis and rheumatoid arthritis

The prevalence of coronary artery atherosclerosis has been found to be increased in patients with chronic inflammatory conditions, such as RA and systemic lupus erythematosus $[3,10]$. Excess mortality due to coronary heart disease in RA patients was first observed in 1953 [11]. In a study of 236 RA patients, the age- and sex-adjusted incidence rate ratio of incident 
Fig. 1 Inflammatory pathway in psoriasis [50]. Working model for immunopathogenesis of psoriasis. Multiple stages are proposed for trafficking patterns of immunocytes, involving signals in which symptomless skin is converted into a psoriatic plaque. Key inflammatory events include intraepidermal trafficking by CD8+ T cells and neutrophils. Reproduced with permission from J Clin Invest (2004, 113:1664-1675). Copyright 2004, The American Society for Clinical Investigation

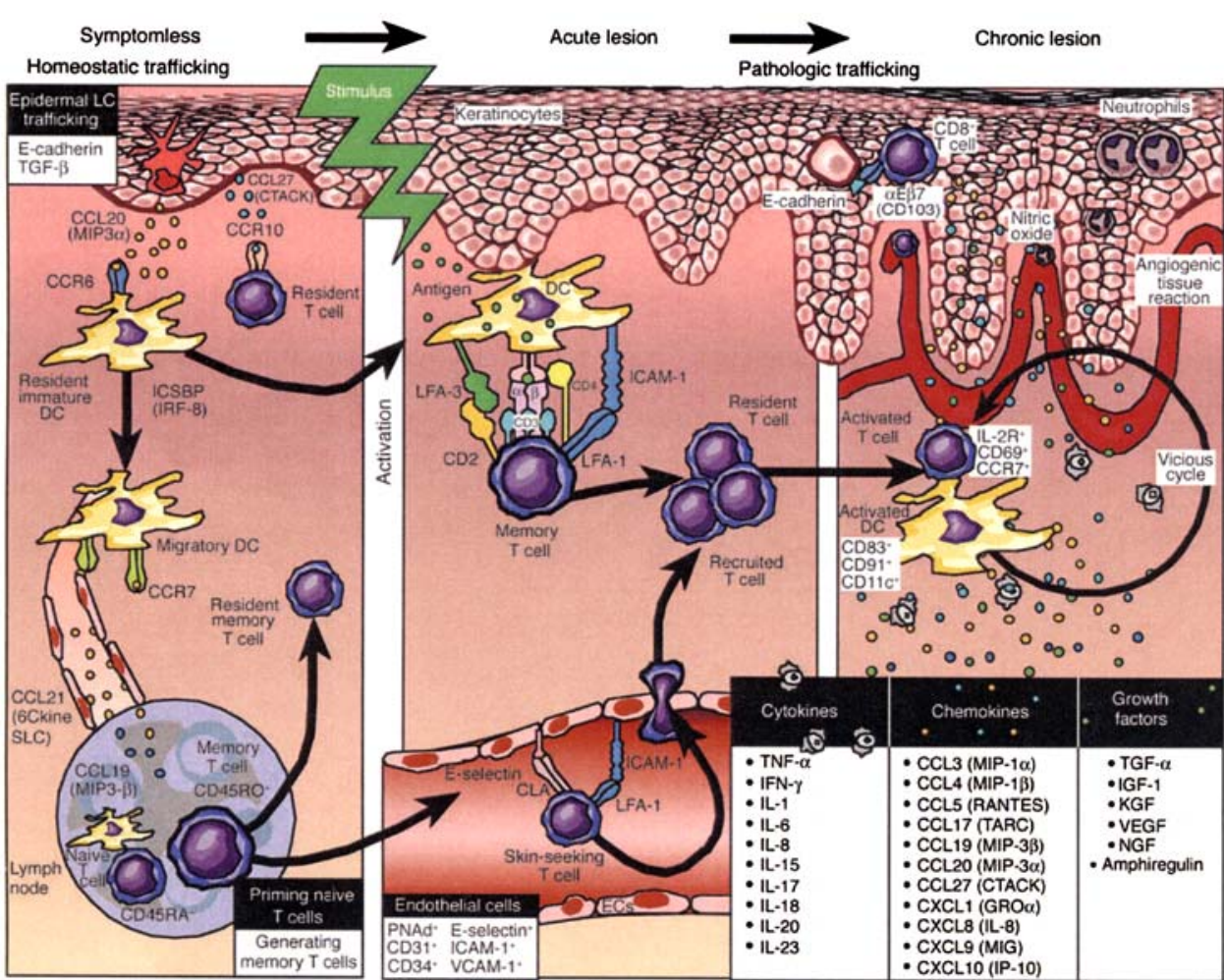

cardiovascular events was calculated to be 3.96 [16]. After adjusting for cardiovascular risk factors such as age, weight and smoking status, this figure decreased slightly to 3.17 , suggesting that there are additional mechanisms responsible for cardiovascular disease in RA. The risk of cardiovascular disease increases with disease severity (eg, number of joints involved) and disease duration $[64,75]$ : severe coronary artery calcification is more likely in patients with established disease ( $>10$ years) compared with controls after adjusting for cardiovascular risk factors (odds ratio $=3.42, P=0.002$ ) [10]. However, there is some emerging evidence for increased atherosclerotic disease in early RA [32].

The lipid profile in RA patients is directly affected by the systemic inflammatory response (active inflammation lowers HDL cholesterol, which leads to increased cardiovascular risk) [75]. Endothelial dysfunction, which underlies atherosclerotic plaque development, is associated with markers of systemic inflammation, such as C-reactive protein (CRP), soluble adhesion molecules (sICAM1 and sE-selectin), IL-6 and TNF-alpha (TNF $\alpha$ ) [24, 38, 78]. Indeed, treatment with the anti-TNF $\alpha$ agent infliximab, which affects expression of all markers described above, has been shown to improve endothelial function in RA patients [28].
Atherosclerosis and psoriasis

An increase in cardiovascular disease has been observed in psoriasis patients [39, 44] and has been shown to be related to disease severity and age of onset $[42,55,62]$. An increased incidence of traditional cardiovascular risk factors is frequently reported $[26,42,56]$. In psoriatic patients with a history of at least one hospital admission due to psoriasis, there is about a $50 \%$ increase in cardiovascular death as compared with the general population [standardised mortality ratio $(\mathrm{SMR})=1.52$. In those patients with 3 or more hospital admissions, the SMR increased to 1.82 and in those patients admitted at younger ages (20-39 years at first admission), the SMR increased to 2.62 [42]. However, the SMR was not increased in those treated as outpatients only, indicating a relationship to disease severity. In patients with PsA, an increase in mortality has also been observed (SMR $=1.65$ in males and 1.59 in females) and the largest single cause of death is myocardial infarction $(27.6 \%)$ [80].

Unfortunately, there is a shortage of published studies corrected for traditional risk factors and no published study has formally assessed systemic vasculature inflammation in psoriasis. 
The increased cardiovascular risk in psoriasis may result from

- The increased prevalence of associated risk factors, such as smoking, obesity, hypertension and alcohol misuse.

- The use of dyslipidaemic therapies, such as corticosteroids, acitretin and ciclosporin.

- An associated unfavourable lipid profile (high triglycerides, low HDL).

- Endothelial dysfunction.

- Uncontrolled inflammation.

- Combination of some or all of the above.

Both psoriasis and atherosclerosis are $\mathrm{T}$ cell-mediated inflammatory conditions and a number of similarities exist between their pathogenetic mechanisms. Numerous factors have been shown to be increased in the serum of psoriatic patients including apolipoprotein E (ApoE), vascular endothelial growth factor (VEGF), sICAM-1, IL-8, soluble interleukin2 receptor (sIL-2R) and TNF $\alpha[9,12,14,15]$. sICAM-1 and sIL$2 \mathrm{R}$ are soluble T-cell products [15]. ApoE plays a central role in lipoprotein metabolism and has been found to be significantly raised in patients with psoriasis, indicating that abnormal metabolism of lipoprotein exists in psoriasis patients [81]. Furthermore, the dyslipidaemic profile exhibited in psoriasis has been shown to modify dendritic cell functions in skin [1]. The impaired dendritic cell emigration from the skin parallels impeded migratory clearance of monocyte-derived dendritic cell-like cells from atherosclerotic plaques.

VEGF mediates endothelial cell mitogenesis and enhances vascular permeability [4]. In psoriasis, VEGF is thought to promote localised angiogenesis and in severe psoriasis may act on renal vasculature to induce hyperpermeability with consequent proteinuria [12]. A role for VEGF has been postulated in the atherosclerotic process as changes in levels of VEGF and its receptors are present in atherosclerosis, and angiogenesis may play a role in plaque development and destabilisation [4].

\section{Genetic basis of comorbidities}

There is considerable evidence for a genetic element in psoriasis. About $30 \%$ of individuals with psoriasis have an affected first-degree relative [69], and first-degree relatives are at a five to tenfold increased risk for developing psoriasis themselves [70]. Concordance rates for psoriasis are three to four times higher in monozygotic twins than dizygotic twins [6], and twin and family studies show that $60-90 \%$ of the variance in psoriasis liability is due to genetic factors. Finally, linkage and association to the major histocompatability complex (MHC) region has been repeatedly demonstrated [58]. However, the presence of many different phenotypes means that not all psoriasis is the same and polygenic inheritance is likely.

Interestingly, by analysing gene polymorphisms of the receptor of advanced glycation end products (RAGE) in patients with plaque-psoriasis seems to be linked to a genetic risk for cardiovascular disorders in psoriasis patients [76].

A combination of disease-specific and inflammatory modifying genes interacting with the environment likely determines the risk of comorbidity. A major susceptibility gene (Psoriasis Susceptibility 1 or PSORS1) is located in the MHC class I region and maps to an approximately $300-\mathrm{kb}$ interval containing HLA-C and 10 other genes in strong linkage disequilibrium with it [25, 47, 77]. Very recently, through a combination of genomic sequencing and haplotype analysis, HLA-Cw6 itself has been identified as the major disease allele at PSORS1 [47].

The observation that bacterial infections are significantly less common in psoriasis patients with an age of onset $\leq 40$ years while immunological skin disorders (allergic contact dermatitis, urticaria and atopic dermatitis) are reduced regardless of age of onset suggests a genetic link between resistance to infection and earlyonset psoriasis [26].

Chronic tonsillitis has been observed over three times more frequently than would be expected in patients with psoriasis [26]. Interestingly, psoriasis is not associated with other forms of streptococcal infection, suggesting a specific role for the tonsils [20]. Recently, identical T-cell receptor rearrangements have been found in the tonsils and lesional skin of psoriatic patients [17]. The prevalence of streptococcal infection during sore throat was 10 times higher in people with psoriasis than in controls and can exacerbate chronic plaque psoriasis [20]. There was, however, no difference in asymptomatic carriage between psoriasis patients and controls [20]. These results suggest either that psoriatic patients are more susceptible to streptococcal infection or that they respond more vigorously to it and are more likely to develop symptoms.

This apparently defective response to streptococci combined with resistance to a variety of skin infections in psoriasis patients provides an interesting paradox. It is known that streptococcal (and other) infections may initiate psoriasis in genetically predisposed individuals, and psoriasis is characterised by strong Th1 immune deviation. Once the psoriatic cascade has been initiated, increased skin and/or systemic inflammation directed by Th1 cells may be responsible for a lower risk of other infections. Interestingly, bacterial infection (principally with Chlamydia pneumoniae) has also 


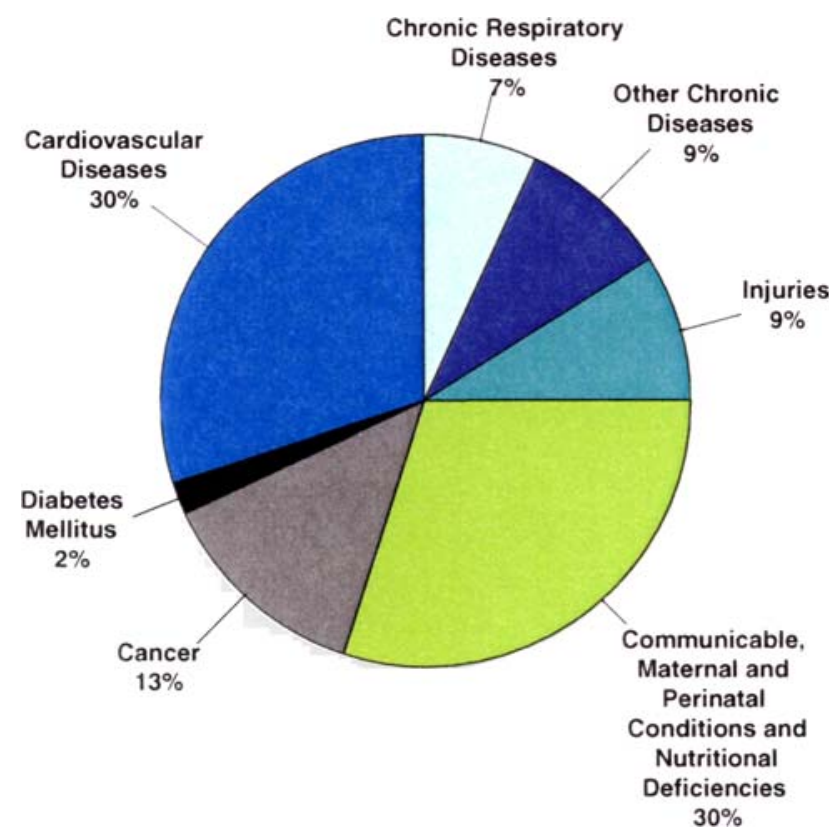

Fig. 2 WHO projected major causes of death 2005 [67]. The occurrence of chronic disease is a rising global burden and accounted for $72 \%$ of the total global burden of disease in the population aged 30 years and older in 2005. Reproduced with permission from Lancet (2005, 366:1578-1582). Copyright 2005, Elsevier Ltd

been implicated in the pathogenesis of atherosclerosis $[36,63]$. A phenomenon similar to that seen in psoriasis could contribute to increased risk of cardiovascular disease.

\section{Clinical importance of comorbidities in psoriasis}

According to the World Health Organization (WHO), the occurrence of chronic disease is a rising global burden. In 2005 , all chronic diseases accounted for $72 \%$ of the total global burden of disease in the population aged 30 years and older (Fig. 2) [68]. More than $80 \%$ of the burden of chronic diseases occurs in people under age 70 years. While psoriasis causes significant morbidity, it is not generally associated with mortality; however, conditions linked with psoriasis are associated with excess mortality [56]. For example, the higher prevalence of smoking in the psoriatic population may explain why psoriasis has been associated with lung cancer in middle-aged women [39]. A registry of 5,687 patients admitted to the hospital for psoriasis between 1973 and 1984 who were followed for up to 22 years until 1995 found a significantly increased mortality for both alcohol- and smoking-related causes [56].

An increased rate of cardiovascular events has been observed in patients with psoriasis [39, 44], and it appears to be related to the severity of disease and age of onset [42, 55, 62]. Worldwide, cardiovascular disease is estimated to be the cause of $30 \%$ of all deaths in 2005 making it the leading single cause of death worldwide [68]. Identification and treatment of modifiable risk factors in patients with psoriasis could result in a lower associated cardiovascular morbidity and mortality [55]. Conceptually, the effective systemic control of inflammation in psoriasis could also lead to improvements in comorbid conditions driven by inflammation.

\section{Implications of comorbidities}

The presence of comorbid conditions in psoriasis patients can have some important implications for clinical management. The general health status of the patient will be affected by all of these associated diseases, with substantial impact on quality of life (QoL) $[33,74]$. The precise impact of comorbid conditions on QoL in psoriasis patients has not been quantified; however, it is known that the presence of joint involvement can significantly reduce health-related QoL [40] and that arthritis and arthralgia are important related factors affecting QoL in patients with psoriasis [83]. In fact, the effects of psoriasis on QoL are comparable to the effects observed from other major chronic diseases [59]. The authors performed an analysis of 317 patients with psoriasis and found that these patients reported a reduction in physical and mental functioning comparable to that observed in patients with cancer, arthritis, hypertension, heart disease and depression [59].

A US study looking at the direct health care expenditures associated with psoriasis found that psoriasis patients treated with systemic therapy or phototherapy have significantly more comorbidities and higher mean total health care expenditures compared with non-psoriasis patients. Furthermore, selected comorbidities lead to significantly higher mean total health care expenditures in those patients with psoriasis than in those with the same condition without psoriasis [13].

Many of the conditions associated with psoriasis could affect the way drugs are absorbed and eliminated from the body. For example, alcoholism impairs liver function, obesity affects drug distribution in the body, diabetes can impair kidney function, inflammatory bowel disease can reduce absorption from the gastrointestinal tract and smoking can impact the efficacy of some drugs [85]. Of course, the presence of these conditions will also lead to increased polypharmacy, increasing the likelihood of drug interactions and making it harder to implement an 'ideal' psoriasis treatment in individuals [34]. 
The implications of managing a patient with comorbid conditions are

- Focus on inflammation control.

- Consider impact on patient as a whole.

- Monitor all associated diseases.

\section{Drug interactions in psoriasis}

It has been suggested that some drugs may trigger or exacerbate psoriasis. The most commonly proposed causative agents are beta blockers, lithium, synthetic antimalarial drugs, nonsteroidal anti-inflammatory drugs (NSAIDs) and tetracycline antibiotics [72]. Analysis of co-medication according to patients' files in 1203 consecutive inpatients hospitalised for treatment of severe psoriasis was conducted between 2003 and 2005 in 9 dermatology departments in Germany [84]. The mean age of patients was 50 years $(55.9 \%$ female, $44.1 \%$ male). The mean Psoriasis Area and Severity Index was 26.0, average body mass index was 27.5 and mean age of first manifestation was 33.5 years. About one third of patients were found to be taking no systemic medication $(34.4 \%)$, and $42.4 \%$ were taking three or fewer systemic agents. However, nearly a quarter $(23.2 \%)$ were taking more than three systemic medications and of these, $11.1 \%$ were taking more than ten medications (Table 2 ). Therefore, physicians caring for patients with psoriasis may consider withdrawal of agents that potentially exacerbate this condition. Further analysis suggests that comorbid cardiac and metabolic disorders are common in these patients: hypertension, $28.8 \%$; type 2 diabetes, $10.5 \%$; dyslipidaemia, $12.5 \%$; hyperuricaemia, $5.3 \%$; arrhythmias, $5.1 \%$; coronary artery disease, $3.2 \%$; and cardiac insufficiency, $2.1 \%$. The presence of renal insufficiency $(2.7 \%)$ and liver cirrhosis $(2.4 \%)$ were also noteworthy.

Table 2 Percentage of psoriasis patients receiving medications for comorbid disease [84]

\begin{tabular}{ll}
\hline Drug class & $\begin{array}{l}\text { Patients }(\%) \\
(n=1,203)\end{array}$ \\
\hline ACE inhibitors & 12.3 \\
Oral anticoagulants & 11.3 \\
Diuretics & 12.4 \\
Thyroid drugs & 9.9 \\
Beta blockers & 7.9 \\
Psycholeptics & 5.6 \\
NSAIDs & 7.0 \\
Lithium salts & 0.70 \\
Interferon-alpha & 0.25 \\
\hline
\end{tabular}

Risk of interactions with psoriasis therapies

There is a high risk of drug interactions with both ciclosporin and methotrexate, while retinoids and leflunomide exhibit a lower risk of drug-drug interactions. Conversely, treatments with biologics, such as TNF $\alpha$ inhibitors, and fumaric acid esters have no known risk of interactions. A number of medications can cause photosensitivity, which is an issue for any UV treatment of psoriasis (e.g., tetracyclines [doxycyclin] and thiazide diuretics) [67].

\section{Effect of psoriasis treatment on comorbidities}

The importance of considering the impact of treatment of diseases associated with psoriasis cannot be stressed enough. Working with physicians in different specialties (e.g., rheumatology, gastroenterology and cardiology) would allow physicians an opportunity to ensure that the psoriatic treatments do not worsen the associated condition and where possible provide additional benefits.

Benefits of psoriasis therapy on comorbid conditions

Since many comorbid conditions have inflammatory mechanisms in common with psoriasis, drugs targeting inflammation and/or suppressing the immune response are often effective in treating both psoriasis and related comorbidities. A number of treatments have shown some efficacy in treating both psoriasis and PsA including methotrexate, ciclosporin, leflunomide, etanercept and infliximab $[2,18,31,45]$. The TNF $\alpha$ inhibitors etanercept and infliximab have demonstrated halting of joint degradation [45].

\section{Crohn's disease}

There are few treatments that are effective in both psoriasis and $\mathrm{CD}$. Azathioprine and 6-mercaptopurine are often used in $\mathrm{CD}$, and they have also shown some efficacy in PsA in small, uncontrolled trials; however, toxicity is likely to restrict their use [50]. Low-dose methotrexate has shown some efficacy in CD patients resistant to other therapies; however, its usefulness in the long term has yet to be established [66]. Infliximab has been shown to be effective in both attaining and maintaining remission in CD patients [23] as well as in the treatment of extra-intestinal cutaneous manifestations [61]. Conversely, etanercept has not been shown to be effective in the treatment of $\mathrm{CD}$ and may even worsen the condition [52]. The difference between the 
effects of TNF $\alpha$ inhibitors on inflammation in CD are thought to be due in part to their different mechanisms of binding TNFa. Infliximab has higher affinity than etanercept, binding more forms of $\mathrm{TNF} \alpha$, and may induce cell apoptosis [35].

\section{Cardiovascular disease}

Evidence for the improvement of psoriasis-associated cardiovascular risk following psoriasis treatment is limited to a trial of methotrexate in patients with psoriasis or RA [57]. Patients prescribed methotrexate had a significantly reduced risk of vascular disease compared with those who did not receive methotrexate (psoriasis, relative risk $[R R]=0.73$; $R A, R R=0.83$ ). Furthermore, risk reduction was greater in those who received a low cumulative dose of methotrexate (psoriasis, $\mathrm{RR}=0.50 ; \mathrm{RA}, \mathrm{RR}=0.65$ ) and in those patients also receiving concomitant folic acid (psoriasis, $\mathrm{RR}=0.56$; $\mathrm{RA}, \mathrm{RR}=0.77$ ). Further work in RA has suggested that anti-TNF $\alpha$ therapy may provide some cardiovascular protection [29]. A comparison of two cohorts, one receiving TNF $\alpha$ inhibitors and one receiving standard therapy (derived from patient registries), revealed a reduced age-sex adjusted incidence rate of first cardiovascular event in those receiving $\mathrm{TNF} \alpha$ inhibitors compared with controls (14.0/1,000 personyears at risk vs. 35.4/1,000 person-years). After controlling for disability, the age-sex adjusted rate ratio was $0.46(P=0.013)$ in the anti-TNF $\alpha$-treated cohort versus the not-treated cohort.

Statins are used to lower lipids in people at risk for cardiovascular events and have been shown to reduce cardiovascular morbidity and mortality; however, statins have also been shown to have anti-inflammatory properties [24, 43]. Statins down-regulate the expression of adhesion molecules and the expression of Th1 chemokine receptors on $\mathrm{T}$ cells, as well as block the expression of $\mathrm{TNF} \alpha$ and interferon-gamma in macrophages among other immunomodulatory actions [49]. A double-blind, placebo-controlled trial in 116 patients with RA demonstrated that $40 \mathrm{mg}$ atorvastatin daily, in addition to existing RA therapy, produced a significant reduction in disease activity $(P=0.004)$, a significant reduction in inflammatory markers (CRP 50\%, $P<0.0001$; ESR $28 \%, P=0.005)$ and a significant reduction in swollen joint count $(-2.69$ vs -0.53 ; $P=0.0058)$ compared with placebo [43]. In addition, simvastatin treatment has been shown to significantly reduce serum CRP and TNF $\alpha$ in patients with RA and improve endothelial function [71]. Therefore, it has been suggested that statins may have beneficial effects in inflammatory dermatological conditions including psoriasis, and the results of controlled trials are awaited [49].

Potential drawbacks of psoriasis therapy on comorbid conditions

Other common comorbidities could influence the choice of psoriasis therapies available to the dermatologist. Ciclosporin can induce hyperglycaemia and acitretin can alter glucose tolerance; therefore, they are best avoided in diabetic patients. Methotrexate has been reported to precipitate diabetes and increase insulin requirements due to increased disease progression [8]. Ciclosporin is contraindicated in patients with uncontrolled hypertension and acitretin is contraindicated in hyperlipidaemia. Methotrexate and acitretin are contraindicated in the presence of hepatic impairment, which may result from psoriasis-associated alcohol misuse, while ciclosporin may need dosage adjustment and regular monitoring of kidney function since renal dysfunction is a known side effect [8].

\section{Drug interactions and precipitating disease in psoriasis}

A diagnosis of psoriasis also has implications regarding treatment for physicians from specialities other than dermatology. The potential of some drugs (eg, angiotensin-converting enzyme inhibitors, beta blockers, NSAIDs) to 'trigger' psoriasis means that these drugs should be avoided where possible and awareness of this potential should be raised. The potential for drug interactions with some psoriasis drugs is high and this should also be considered when designing therapeutic regimens. Furthermore, the increased risk of cardiovascular events associated with psoriasis should be considered when conducting global cardiovascular risk assessments. If possible, lower cut-off points for treatment should be used in psoriasis patients in an effort to take into consideration their overall cardiovascular risk.

\section{Discussion and conclusions}

Psoriasis is a multifaceted systemic condition that is not restricted to cutaneous symptoms. There is a need for further research to fully understand the reasons for comorbidities and their implications on treatment and management of psoriasis patients. Data are required to fully characterise risk of cardiovascular disease in psoriasis patients and whether treatment can impact this level of risk. Could effective (systemic) treatment of psoriasis reduce the risk of comorbidities or, conversely, 
could effective treatment of comorbidities improve psoriasis? Investigation of the genetics of psoriasis and related inflammatory conditions may provide insight into their pathogenesis and points of commonality, which could reveal novel drug targets

However, while research is conducted, there is a pressing need for education about comorbidities in psoriasis. Dermatologists need to be aware of the likelihood of comorbid conditions to ensure their detection and that psoriasis is managed with consideration of the possible impact on associated comorbid inflammatory conditions. Similarly, physicians from other specialities should be aware of the potential impact of their management strategies on psoriasis and take care not to exacerbate psoriasis when treating associated conditions. A focus on rapid and profound systemic control of inflammation in psoriasis may have added benefits by controlling inflammation associated with comorbid conditions. It is clear that TNF $\alpha$ is a central mediator in psoriasis and many of the recognised associated diseases. Knowledge and awareness of the common inflammatory processes involved should provide the opportunity to more effectively treat the common causes of inflammatory conditions benefitting both physicians and patients. Managing the psoriasis patient as a whole with integrated therapy is likely to maximise outcomes.

\section{References}

1. Angeli V, Llodra J, Rong JX et al (2004) Dyslipidemia associated with atherosclerotic disease systemically alters dendritic cell mobilization. Immunity 21:561-574

2. Antoni CE, Kavanaugh A, Kirkham B et al (2005) Sustained benefits of infliximab therapy for dermatologic and articular manifestations of psoriatic arthritis: results from the infliximab multinational psoriatic arthritis controlled trial (IMPACT). Arthritis Rheum 52:1227-1236

3. Asanuma Y, Oeser A, Shintani AK et al (2003) Premature coronary-artery atherosclerosis in systemic lupus erythematosus. N Engl J Med 349:2407-2415

4. Belgore F, Blann A, Neil D et al (2004) Localisation of members of the vascular endothelial growth factor (VEGF) family and their receptors in human atherosclerotic arteries. J Clin Pathol 57:266-272

5. Bernstein CN, Wajda A, Blanchard JF (2005) The clustering of other chronic inflammatory diseases in inflammatory bowel disease: a population-based study. Gastroenterology 129:827-836

6. Bhalerao J, Bowcock AM (1998) The genetics of psoriasis: a complex disorder of the skin and immune system. Hum Mol Genet 7:1537-1545

7. Bowcock AM, Krueger JG (2005) Getting under the skin: the immunogenetics of psoriasis. Nat Rev Immunol 5:699-711

8. Buckingham BA, Sandborg CI (2000) A randomized trial of methotrexate in newly diagnosed patients with type 1 diabetes mellitus. Clin Immunol 96:86-90
9. Campalani E, Allen MH, Fairhurst D et al (2006) Apolipoprotein $\mathrm{E}$ gene polymorphisms are associated with psoriasis but do not determine disease response to acitretin. Br J Dermatol 154:345-352

10. Chung CP, Oeser A, Raggi P et al (2005) Increased coronaryartery atherosclerosis in rheumatoid arthritis: relationship to disease duration and cardiovascular risk factors. Arthritis Rheum 52:3045-3053

11. Cobb S, Anderson F, Bauer W (1953) Length of life and cause of death in rheumatoid arthritis. N Engl J Med 249:553-556

12. Creamer D, Allen M, Jaggar R et al (2002) Mediation of systemic vascular hyperpermeability in severe psoriasis by circulating vascular endothelial growth factor. Arch Dermatol 138:791-796

13. Crown WH, Bresnahan BW, Orsini LS et al (2004) The burden of illness associated with psoriasis: cost of treatment with systemic therapy and phototherapy in the US. Curr Med Res Opin 20:1929-1936

14. De Pita O, Ruffelli M, Cadoni S et al (1996) Psoriasis: comparison of immunological markers in patients with acute and remission phase. J Dermatol Sci 13:118-124

15. De Rie MA, Zonneveld IM, Witkamp L et al (1996) Soluble interleukin-2 receptor (sIL-2R) is a marker of disease activity in psoriasis: a comparison of sIL-2R, sCD27, sCD4, sCD8 and sICAM-1. Acta Derm Venereol 76:357-360

16. Del Rincon ID, Williams K, Stern MP et al (2001) High incidence of cardiovascular events in a rheumatoid arthritis cohort not explained by traditional cardiac risk factors. Arthritis Rheum 44:2737-2745

17. Diluvio L, Vollmer S, Besgen P et al (2006) Identical TCR beta-chain rearrangements in streptococcal angina and skin lesions of patients with psoriasis vulgaris. J Immunol 176:7104-7111

18. Fraser AD, van Kuijk AW, Westhovens R et al (2005) A randomised, double blind, placebo controlled, multicentre trial of combination therapy with methotrexate plus ciclosporin in patients with active psoriatic arthritis. Ann Rheum Dis 64:859-864

19. Gisondi P, Girolomoni G, Sampogna F et al (2005) Prevalence of psoriatic arthritis and joint complaints in a large population of Italian patients hospitalised for psoriasis. Eur J Dermatol 15:279-283

20. Gudjonsson JE, Thorarinsson AM, Sigurgeirsson B et al (2003) Streptococcal throat infections and exacerbation of chronic plaque psoriasis: a prospective study. Br J Dermatol 149:530-534

21. Gupta MA, Gupta AK (1998) Depression and suicidal ideation in dermatology patients with acne, alopecia areata, atopic dermatitis and psoriasis. Br J Dermatol 139:846-850

22. Gupta MA, Gupta AK (2003) Psychiatric and psychological co-morbidity in patients with dermatologic disorders: epidemiology and management. Am J Clin Dermatol 4:833-842

23. Hanauer SB, Feagan BG, Lichenstein GR et al (2002) Maintenance infliximab for Crohn's disease: the ACCENT I randomised trial. Lancet 359:1541-1549

24. Hansson GK (2005) Inflammation, atherosclerosis and coronary artery disease. N Engl J Med 352:1685-1695

25. Helms C, Saccone NL, Cao L et al (2005) Localization of PSORS1 to a haplotype block harbouring HLA-C and distinct from corneodesmosin and HCR. Hum Genet 118:466476

26. Henseler T, Christophers E (1995) Disease concomitance in psoriasis. J Am Acad Dermatol 32:982-986

27. Herron MD, Hinckley M, Hoffman MS et al (2005) Impact of obesity and smoking on psoriasis presentation and management. Arch Dermatol 141:1527-1534 
28. Hurlimann D, Forster A, Noll G et al (2002) Anti-tumor necrosis factor-alpha treatment improves endothelial function in patients with rheumatoid arthritis. Circulation 106:2184-2187

29. Jacobsson LTH, Turesson C, Gulfe A et al (2005) Treatment with tumor necrosis factor blockers is associated with a lower incidence of first cardiovascular events in patients with rheumatoid arthritis. J Rheumatol 32:1213-1218

30. Jones SM, Harris CPD, Lloyd J et al (2000) Lipoproteins and their subfractions in psoriatic arthritis: identification of an atherogenic profile with active joint disease. Ann Rheum Dis 59:904-909

31. Kaltwasser JP, Nash P, Gladman D et al (2004) Efficacy and safety of leflunomide in the treatment of psoriatic arthritis and psoriasis: a multinational, double-blind, randomized, placebo-controlled clinical trial. Arthritis Rheum 50:19391950

32. Kaplan M, McCune WJ (2003) New evidence for vascular disease in patients with early rheumatoid arthritis. Lancet 361:1068-1069

33. Kimball AB, Jacobson C, Weiss S et al (2005) The psychosocial burden of psoriasis. Am J Clin Dermatol 6:383-392

34. Kohler GI, Bode-Boger SM, Busse R et al (2000) Drug-drug interactions in medical patients: effects of in-hospital treatment and relation to multiple drug use. Int J Clin Pharmacol Ther 38:504-513

35. Kruger-Krasagakis S, Galanopoulos VK, Giannikaki L, Stefanidou M, Tosca AD (2006) Programmed cell death of keratinocytes in infliximab-treated plaque-type psoriasis. $\mathrm{Br} \mathbf{J}$ Dermatol 154:460-466

36. Kuo C-C, Campbell LA (2000) Detection of Chlamydia pneumoniae in arterial tissues. J Infec Dis 181:S432-S436

37. Lee FJ, Bellary SV, Francis C (1990) Increased occurrence of psoriasis in patients with Crohn's disease and their relatives. Am J Gastroenterol 85:962-963

38. Libby P, Ridker PM (2004) Inflammation and atherosclerosis: role of C-reactive protein in risk assessment. Am J Med 116:9S-16S

39. Lindegard B (1986) Diseases associated with psoriasis in a general population of 159,200 middle-aged, urban, native Swedes. Dermatologica 172:298-304

40. Lundberg L, Johannesson M, Silverdahl M et al (2000) Health-related quality of life in patients with psoriasis and atopic dermatitis measured with SF-36, DLQI and a subjective measure of disease activity. Acta Derm Venereol 80:430 434

41. Madland TM, Apalset EM, Johannessen AE, Rossebo B, Brun JG (2005) Prevalence, disease manifestations, and treatment of psoriatic arthritis in Western Norway. J Rheumatol 32:1918-1922

42. Mallbris L, Akre O, Granath F et al (2004) Increased risk for cardiovascular mortality in psoriasis inpatients but not in outpatients. Eur J Epidemiol 19:225-230

43. McCarey DW, McInnes IB, Madhok R et al (2004) Trial of atorvastatin in rheumatoid arthritis (TARA): double-blind, randomised placebo-controlled trial. Lancet 363:2015-2021

44. McDonald CJ, Calabresi P (1978) Psoriasis and occlusive vascular disease. Br J Dermatol 99:469-475

45. Mease PJ, Kivitz AJ, Burch FX et al (2004) Etanercept treatment of psoriatic arthritis: safety, efficacy, and effect on disease progression. Arthritis Rheum 50:2264-2272

46. Morse RM, Perry HO, Hurt RD (1985) Alcoholism and psoriasis. Alcohol Clin Exp Res 9:396-399

47. Nair RP, Stuart PE, Nistor I et al (2006) Sequence and haplotype analysis supports HLA-C as the psoriasis susceptibility 1 gene. Am J Hum Genet 78:827-851
48. Naldi L, Chatenoud L, Linder D et al (2005) Cigarette smoking, body mass index, and stressful life events as risk factors for psoriasis: results from an Italian case-control study. J Invest Dermatol 125:61-67

49. Namazi MR (2004) Statins: novel additions to the dermatologic arsenal? Exp Dermatol 13:337-339

50. Nash P, Clegg DO (2005) Psoriatic arthritis therapy: NSAIDs and traditional DMARDs. Ann Rheum Dis 64:ii74-ii77

51. Nickoloff BJ, Nestle FO (2004) Recent insights into the immunopathogenesis of psoriasis provide new therapeutic opportunities. J Clin Invest 113:1664-1675

52. Oh J, Arkfeld DG, Horwitz DA (2005) Development of Crohn's disease in a patient taking etanercept. J Rheumatol 32:752-753

53. Pearce DJ, Morrison AE, Higgins KB et al (2005) The comorbid state of psoriasis patients in a university dermatology practice. J Derm Treat 16:319-323

54. Persson PG, Leijonmarck CE, Bernell O et al (1993) Risk indicators for inflammatory bowel disease. Int $\mathrm{J}$ Epidemiol 22:268-272

55. Peters MJ, van der Horst-Bruinsma IE, Dijkmans BA, Nurmohamed MT (2004) Cardiovascular risk profile of patients with spondyloarthropathies, particularly ankylosing spondylitis and psoriatic arthritis. Semin Arthritis Rheum 34:585592

56. Poikolainen K, Karvonen J, Pukkal E (1999) Excess mortality related to alcohol and smoking among hospital-treated patients with psoriasis. Arch Dermatol 135:1490-1493

57. Prodanowich S, Ma F, Taylor JR et al (2005) Methotrexate reduces incidence of vascular diseases in veterans with psoriasis or rheumatoid arthritis. J Am Acad Dermatol 52:262-267

58. Rahman P, Elder JT (2005) Genetic epidemiology of psoriasis and psoriatic arthritis. Ann Rheum Dis 64:ii37-ii39

59. Rapp SR, Feldman SR, Exum ML, Fleischer AB Jr, Beboussin DM (1999) Psoriasis causes as much disability as other major medical diseases. J Am Acad Dermatol 41(3 Pt 1):401407

60. Reynoso-von Drateln C, Martinez-Abundis E, BalcazarMunoz BR et al (2003) Lipid profile, insulin secretion, and insulin sensitivity in psoriasis. J Am Acad Dermatol 48:882885

61. Rispo A, Scarpa R, Di Girolamo E et al (2005) Infliximab in the treatment of extra-intestinal manifestations of Crohn's disease. Scand J Rheumatol 34:387-391

62. Rocha-Pereira P, Santos-Silva A, Rebelo I, et al (2001) Dislipidemia and oxidative stress in mild and in severe psoriasis as a risk for cardiovascular disease. Clin Chim Acta 303:33-39

63. Saikku P, Leinonen M, Mattila K et al (1988) Serological evidence of an association of a novel Chlamydia, TWAR, with chronic coronary heart disease and acute myocardial infarction. Lancet 2:983-986

64. Sattar N, McInnes IB (2005) Vascular comorbidity in rheumatoid arthritis: potential mechanisms and solutions. Curr Opin Rheumatol 17:286-292

65. Schmitt D, Carstensen K, Bugdahl R, Christophers E, Weichenthal M (2003) The association of psoriasis vulgaris with internal diseases. Arch Dermatol Res 294:487

66. Schroder O, Stein J (2003) Low dose methotrexate in inflammatory bowel disease: current status and future directions. Am J Gastroenterol 98:530-537

67. Stern RS, Kleinerman RA, Parrish JA et al (1980) Phototoxic reactions to photoactive drugs in patients treated with PUVA. Arch Dermatol 116:1269-1271

68. Strong K, Mathers C, Leeder S, Beaglehole R (2005) Preventing chronic diseases: how many lives can we save? Lancet 366:1578-1582 
69. Swanbeck G, Inerot A, Martinsson T, Wahlstrom J (1994) A population genetic study of psoriasis. Br J Dermatol 131:32-39

70. Swanbeck G, Inerot A, Martinsson T et al (1997) Genetic counselling in psoriasis: empirical data on psoriasis among first-degree relatives of 3095 psoriatic probands. Br J Dermatol 137:939-942

71. Tikiz C, Utuk O, Pirildar T et al (2005) Effects of angiotensinconverting enzyme inhibition and statin treatment on inflammatory markers and endothelial functions in patients with longterm rheumatoid arthritis. J Rheumatol 32:2095-2101

72. Tsankov N, Angelova I, Kazandjieva J (2000) Drug-induced psoriasis. Recognition and management. Am J Clin Dermatol 1:159-165

73. Uyanik BS, Ari Z, Onur E et al (2002) Serum lipids and apolipoproteins in patients psoriasis. Clin Chem Lab Med 40:6568

74. Van de Kerkhof P et al. Available at: http://www.psori.fi/doc/ patient_survey_2nd_phase(2003).pdf. Accessed September 15,2005

75. Van Doornum S, McColl G, Wicks IP (2002) Accelerated atherosclerosis: an extraarticular feature of rheumatoid arthritis? Arthritis Rheum 46:862-873

76. Vasku V, Kankova K, Vasku A et al (2002) Gene polymorphisms (G82S, 1704G/T, 2184A/G and 2245G/A) of the receptor of advanced glycation end products (RAGE) in plaque psoriasis. Arch Dermatol Res 294:127-1230

77. Veal CD, Capon F, Allen MH et al (2002) Family-based analysis using a dense single-nucleotide polymorphism-based map defines genetic variation at PSORS1, the major psorisasis-susceptibility locus. Am J Hum Genet 71:554-564
78. Wallberg-Jonsson S, Cvetkovic JT, Sundqvist K-G et al (2002) Activation of the immune system and inflammatory activity in relation to markers of atherothrombotic disease and atherosclerosis in rheumatoid arthritis. J Rheumatol 29:875-882

79. Williams JP, Meyers JA (2002) Immune-mediated inflammatory disorders (I.M.I.D.s): the economic and clinical costs. Am J Manag Care 8:S664-S681

80. Wong K, Gladman DD, Husted J et al (1997) Mortality studies in psoriatic arthritis: results from a single outpatient clinic. I. Causes and risk of death. Arthritis Rheum 40:1868-1872

81. Xiao T, Yang C, Xiao Y, Song F (1997) Serum apolipoprotein levels of psoriatic patients with normal serum lipid levels. Chin Med Sci J 12:224-228

82. Yates VM, Watkinson G, Kelman A (1982) Further evidence for an association between psoriasis, Crohn's disease and ulcerative colitis. Br J Dermatol 106:323-330

83. Zachariae H, Zachariae R, Blomqvist K et al (2002) Quality of life and prevalence of arthritis reported by 5,795 members of the Nordic Psoriasis Associations. Data from the Nordic Quality of Life Study. Acta Derm Venereol 82:108-113

84. Zahl V, Gerdes S, Mrowietz U Co-medication in patients with severe psoriasis: first results of a retrospective analysis in 1203 hospitalized patients in Germany. Presented at the 4th International Congress-The Royal College of Physicians, December 1-3, 2005, London, P-67

85. Zevin S, Benowitz NL (1999) Drug interactions with tobacco smoking. An update. Clin Pharmacokinet 36:425-438

86. Zimmet P, Maglano D, Matsuzawa Y et al (2005) The metabolic syndrome: a global public health problem and a new definition. J Atheroscler Thromb 12:295-300 